\title{
Optical pumping of helium with arc lamp excited LNA lasers
}

\author{
C. G. Aminoff $(*)$, C. Larat, M. Leduc and F. Laloë \\ Laboratoire de Spectroscopie Hertzienne de l'Ecole Normale Supérieure, 24 rue Lhomond, \\ 75231 Paris Cedex 05, France
}

(Reçu le 8 avril 1989, accepté le 9 mai 1989)

\begin{abstract}
Résumé. - Nous décrivons un laser compact et relativement bon marché qui peut servir de source pour le pompage optique de l'hélium ; il fonctionne à la longueur d'onde dans l'infrarouge proche qui correspond à la transition du métastable triplet vers le premier niveau $P$ supérieur. Le milieu amplificateur est un cristal de LNA pompé transversalement par des lampes à arc à krypton. La puissance totale dépasse $800 \mathrm{~mW}$ à $\lambda=1,083 \mu \mathrm{m}$, avec une largeur spectrale de l'ordre de $3 \mathrm{GHz}$. Une polarisation nucléaire de $66 \%$ a pu être obtenue de cette façon dans un gaz de ${ }^{3} \mathrm{He}$.
\end{abstract}

\begin{abstract}
We have developed a compact and relatively inexpensive laser source at $\lambda=1.083 \mu \mathrm{m}$, suitable for optical pumping in helium. The laser is made of an LNA crystal transversely pumped by krypton arc lamps. A cw power of $800 \mathrm{~mW}$ has been achieved at $1.083 \mu \mathrm{m}$ with a $3 \mathrm{GHz}$ bandwidth. A $66 \%$ nuclear polarization has been obtained in a ${ }^{3} \mathrm{He}$ gas.
\end{abstract}

Applying the optical pumping method to the $2^{3} \mathrm{~S}_{1}$ metastable level of helium-4, populated in a weak gaseous discharge, is an idea dating back to 1960 [1]. The electronic polarization obtained in this way can be used for various purposes, including the design of sensitive magnetometers [2]. A few years later, Colegrove, Schearer and Walters realized that, when applied to the light isotope helium-3, the same method can provide a substantial nuclear polarization of the atoms in their $1^{1} \mathrm{~S}_{0}$ ground state, under the effect of the metastability exchange collisions between the $2^{3} S_{1}$ and $1^{1} S_{0}[3,4]$. Several applications of these techniques were found and developed [5], but a constant limitation was the relatively small intensity of the discharge lamps used as optical pumping sources; this puts an upper limit of the order of $20 \%$ on the nuclear polarizations achievable by the method.

The situation changed when lasers operating at $\lambda=1.083 \mu \mathrm{m}$ (the wavelength of the $2^{3} \mathrm{~S}-2^{3} \mathrm{P}$ transition of helium) became available, allowing to obtain higher nuclear polarisations [6] than before.

(*) Permanent address : Department of Technical Physics, Helsinki University of Technology, SF-02150 ESPOO, Finland.
This renewed the interest in the applications of optical pumping in helium to various fields of physics, namely studies of quantum fluids at low temperature [7], polarized helium-3 targets for nuclear physics experiments [8] with enhanced densities either by low temperature techniques $[9,10]$ or by mechanical compression [11], spin state filters for neutron beams [12], fusion reactor plasmas with polarized nuclei [13] or sources of spin polarized electrons using optically pumped helium-4 metastable atoms [14].

For all these applications, especially if one plans the production of larger amounts of spin polarized gas which will require even higher pumping powers, it is desirable to improve further the characteristics of the laser sources suitable for helium optical pumping. The laser should be tunable around $\lambda=1.08 \mu \mathrm{m}$, continuous (to give more efficient optical pumping by reducing optical saturation : the optical pumping cycle becomes ineffective if stimulated emission replaces spontaneous emission), sufficiently easy to handle to be used in the environment of particle accelerators, and of course powerful enough - the intensity of the laser being a limiting factor in several cases. Among the techniques developed for this purpose, generally based on new solid 
state materials, the LNA laser soon appeared as a particularly useful tool for several laboratory experiments [15]. LNA crystals of small dimensions were pumped longitudinally with $\mathrm{Ar}^{+}$or $\mathrm{Kr}^{+}$lasers [16] or with diode arrays [17] and they showed laser emission with a broad tuning range and a fairly high efficiency at $1.083 \mu \mathrm{m}$. However, the power was still limited and, furthermore, the cost and lack of reliability of ion-gas lasers used for the pumping of LNA raised additional problems.

It was therefore tempting to try laser techniques similar to those used for Nd: YAG lasers, which can deliver very high $\mathrm{CW}$ powers (several hundreds of watts) at $1.064 \mu \mathrm{m}$, a wavelength not absorbed by helium atoms but not very far from the desired value at $1.083 \mu \mathrm{m}$. Such high powers are currently achieved by transverse pumping of long rods by continuous arc lamps filled with noble gases. Some interesting results for helium orientation were obtained previously with this kind of technique, not with LNA, but with Nd: YAP crystals [18]. However, the helium wavelength is situated far on the wing of one of the Nd : YAP emission bands, which puts practical limitations on the available power and makes the laser somewhat difficult to operate. It therefore seemed attractive, as soon as sufficiently long LNA crystals became available, to pump them with arc lamps in a YAG type cavity. Recently, encouraging preliminary results in this direction have been obtained by Milner et al. [19] and communicated to us privately. The purpose of this article is to report the results of LNA laser operation that we have obtained, as well as the demonstration of substantial nuclear polarizations in a gas of helium-3 with this laser.

\section{The lamp pumped LNA laser.}

LNA is a neodymium laser material in many respects similar to Nd:YAG. LNA stands for $\mathrm{La}_{1-x} \mathrm{Nd}_{x} \mathrm{MgAl}_{11} \mathrm{O}_{19}$, where $x$ is usually between 0.1 and 0.2 . This material offers some advantages over Nd: YAG, such as the possibility of high doping concentration ; on the other hand, several difficulties arise for using LNA in a lamp pumped laser cavity. First, the growth of single crystals with good optical quality is not easy ; in particular, long rods cut along the $c$-axis, which is the best for the laser action efficiency [16], are not yet available. Second, the thermal conductivity of the lattice is poor, 2 or 3 times smaller than for Nd:YAG [20]. This gives rise to large temperature gradients between the center and the outside of the rod, and consequently to strong thermal focussing effects of the crystals inside the laser resonator [21], even at moderate power of the lamp excitation. Furthermore, the LNA material is strongly anisotropic. Many physical properties, such as the thermoconductivity and dila- tation coefficient, are different along the $c$-axis and along any direction perpendicular to the $c$-axis [20]. This can result in some optical astigmatism under the effect of anisotropic thermal focussing. It can also increase the risk of breaking the crystal under lamp heating, because of the distorsion and stress thermally induced in the different transverse directions.

The size of the LNA rod (purchased from Union Carbide) was $95 \mathrm{~mm}$ in length and $5 \mathrm{~mm}$ in diameter. It was cut along the $a$-axis and doped to $15 \%$ of neodymium. The rod was excited with two krypton discharge lamps in the laser head of a Micro-Contrôle YAG-904 cavity. For designing the resonator, we used the computer program developed earlier [22] which gives the stability zones and the beam waist size inside a cavity containing a long focussing element. However, this calculation could only be taken as a guide, because it includes simplified assumptions on the transverse mode of the beam (supposed to be $\mathrm{TEM}_{00}$ ) and on the focussing rod (supposed to be transversely isotropic). The solution that we chose is probably not unique but we found it convenient because the stability domain is relatively large around a medium power of the lamps; moreover there is an internal beam waist inside the cavity, which can be useful for the insertion of selective elements. The laser cavity is shown schematically in figure 1 . The ends of the LNA rods are anti-reflection coated. They are curved with a $60 \mathrm{~cm}$ radius, in order to partially compensate the thermal focussing effect. The crystal is oriented so that its $c$-axis is vertical. Mirror $\mathrm{M}_{1}$ is plane and has a high reflectivity of $99.9 \%$. Mirror $\mathbf{M}_{2}$ is concave, with a $50 \mathrm{~cm}$ radius and a reflectivity of $99.5 \%$; it is used as the output coupler. Distances between the rod and the mirrors are indicated in the figure but were not found to be critical within a few centimeters.

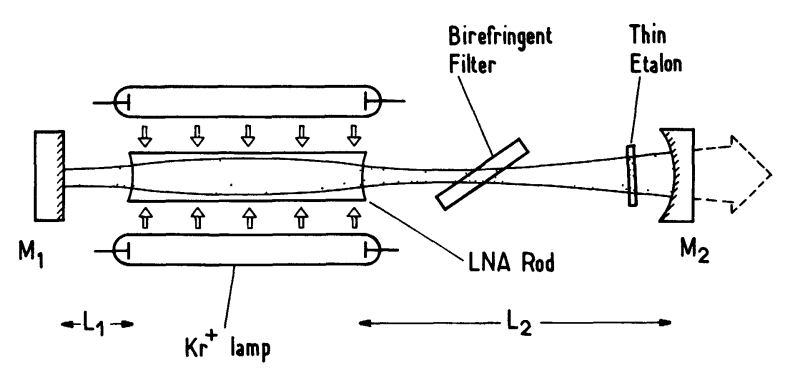

Fig. 1. - Scheme of the L.N.A. laser cavity (not to scale). The resonator is formed by a plane high-reflecting end mirror $M_{1}$ and a spherical output coupler $M_{2}$ with a $50 \mathrm{~cm}$ radius of curvature. The mirrors are at the distances $L_{1}=10 \mathrm{~cm}$ and $L_{2}=50 \mathrm{~cm}$ from the rod ends. The end faces of the rod are curved with a radius of $60 \mathrm{~cm}$. A single-plate birefringent filter and a thin etalon are used for line narrowing and fine tuning to the helium wavelength. The effect of thermal focussing on the beam shape is schematically indicated. 


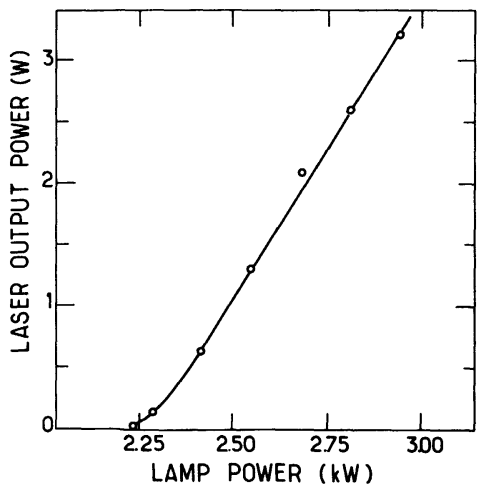

Fig. 2. - Output power of the lamp pumped LNA laser (without selective elements) as a function of the electrical power of the $\mathrm{Kr}^{+}$arc lamps. An output coupler with $0.5 \%$ transmission was used. With no selective optics, the laser emitted at $1.08 \mu \mathrm{m}$ and $1.05 \mu \mathrm{m}$ simultaneously.

Figure 2 shows the output power measured without any selective elements inside the cavity. The relatively high value of the threshold can be attributed to the fact that, when the excitation power is low, the thermal focussing effects are not sufficient to compensate the lens effects introduced by the curved ends of the rod, so that the resonator falls outside the domain of stability. This high value might also partially result from the losses inside the rod, which we attempted to evaluate using an auxiliary laser beam at $1.083 \mu \mathrm{m}$ that we passed through the crystal. The losses were found to be less than $2 \%$, but could not be accurately measured. With this rod we observed that the gain is nearly the same on the two bands around 1.05 and $1.08 \mu \mathrm{m}$. Wavelength tuning was obtained by inserting a single plate birefringent filter $(6 \mathrm{~mm}$ thick) at the beam waist location inside the cavity. The laser polarization was spontaneously fixed by the orientation of the crystal, parallel to the $a^{*}$ axis. The position of the birefringent filter was carefully adjusted to meet a Brewster angle condition for this polarization. Figure 3 shows the tuning bands ob-

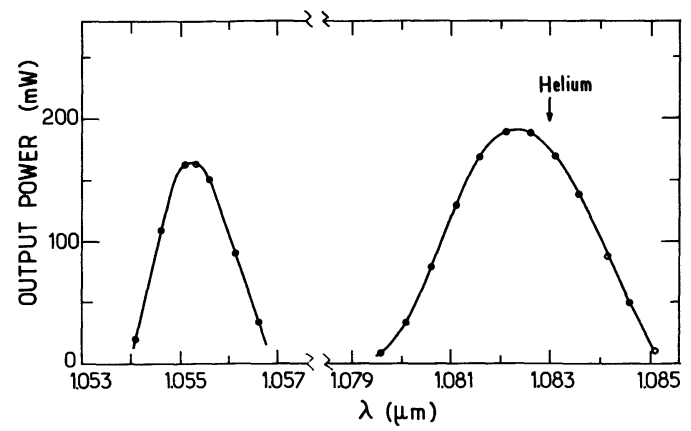

Fig. 3. - Tuning curve of the lamp pumped LNA laser obtained with a lamp excitation power of $2.7 \mathrm{~kW}$. A single-plate birefringent filter with a thickness of $6 \mathrm{~mm}$ was used for tuning. The wavelength of the $2{ }^{3} \mathrm{~S}-2{ }^{3} \mathrm{P}$ transition in helium at $1.083 \mu \mathrm{m}$ is indicated. served. They are slightly narrower than for pumping with a gas laser or a diode laser, probably because the arc-lamp pumped laser operates closer to threshold.

The set of selective elements that we found best suited for the optical pumpping experiments are those shown in figure 1 . The birefringent filter is a $2 \mathrm{~mm}$ thick quartz plate, which can completely suppress lasing on the $1.05 \mu \mathrm{m}$ band and allows tuning to the helium wavelength. In addition, we use a solid etalon, $0.7 \mathrm{~mm}$ thick, made of fused silica, for line narrowing and fine tuning. At the helium frequency, we then reached an output power of $870 \mathrm{~mW}$ for pumping with lamps at $3 \mathrm{~kW}$ electrical power. The spectral width of this laser was measured with a plane Fabry-Perot analyzer and found to be of the order of $3 \mathrm{GHz}$ (FWHM). The mode structure consists of a large number of transverse modes within the measured width. Another indication of the frequency spread is given by the fluorescence curves registered using a ${ }^{3} \mathrm{He}$ cell irradiated with this laser : figure 4 shows a recording obtained by sweeping the laser frequency over the structure of the $2^{3} \mathrm{~S}-$ $2{ }^{3} \mathrm{P}$ transition by mechanically tilting the thin etalon

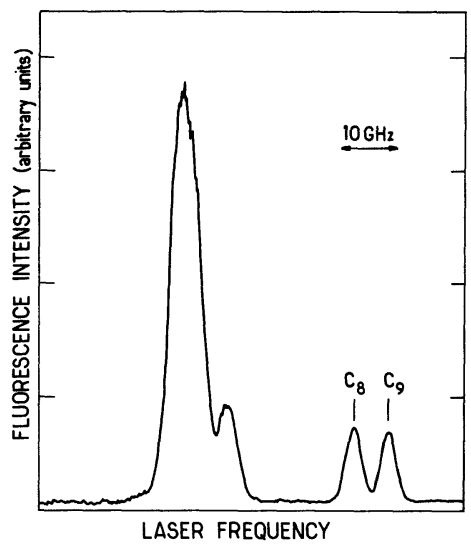

Fig. 4. - Spectrum of ${ }^{3} \mathrm{He}$ around $\lambda=1.083 \mu \mathrm{m}$ recorded by tuning the LNA laser frequency (cf. Fig. 1) and detecting the fluorescence of the gas. The pressure in the cell was 0.3 torr. The curve shows the structure of the $2{ }^{3} \mathrm{~S}_{1}-2{ }^{3} \mathrm{P}$ transition in ${ }^{3} \mathrm{He}$ and the two components $\mathrm{C}_{8}$ and $\mathrm{C}_{9}$, corresponding to the $\left(2{ }^{3} \mathrm{~S}_{1}, F=1 / 2 \rightarrow 2{ }^{3} \mathrm{P}_{0}\right)$ and $\left(2^{3} \mathrm{~S}_{1}, F=3 / 2 \rightarrow 2{ }^{3} \mathrm{P}_{0}\right)$ transitions, are clearly resolved.

shown in figure 1 . The two components $\mathrm{C}_{8}$ and $\mathrm{C}_{9}$ (notations of Ref. [5]), 6.6 GHz apart (hyperfine structure of the $2^{3} \mathrm{~S}$ level), appear well separated and their width is of the order of the Doppler broadening of the atoms ; this shows that the spectral width of the laser does not much exceed $2 \mathrm{GHz}$, a value that very well fits the purpose of optical pumping over the entire Doppler width of the helium atoms. 


\section{Nuclear polarization of helium-3.}

The laser was then used to optically pump a ${ }^{3} \mathrm{He}$ gas. The helium container was a cylinder, $5 \mathrm{~cm}$ in diameter and $5 \mathrm{~cm}$ in length, in which a light R.F. discharge was sustained. The laser output is spontaneously almost totally linearly polarized (see above), but better results were obtained by improving the purity of the polarization with a Glan Thompson polarizer (external to the laser cavity). The circular polarization was introduced by the addition of a plastic quarter-wave plate (Polaroïd $280 \mathrm{~m} \mu$ ). The laser beam is divergent and its size on the helium cell, located $1.5 \mathrm{~m}$ away, was about $4 \mathrm{~cm}$ in diameter, which we found optimum for directly exciting most of the metastable atoms in the cell. The beam was reflected back into the cell by a metallic mirror. The degree of nuclear polarization was measured using an optical method, through the measurement of the circular polarization of the $\lambda=668 \mathrm{~nm}$ line emitted by the discharge [23]. The magnetic field was $5 \mathrm{G}$ and its homogeneity was mostly limited by the laboratory environment ; the corresponding relaxation time $T_{1}$, with discharge off, was of the order of $10 \mathrm{~min}$.

Figure 5 shows the nuclear polarization measured as a function of the LNA laser intensity for a helium3 pressure of 0.3 torr. Very comparable values were obtained with another cell containing a 1 torr pressure. Each measurement was made after adjusting the laser frequency on the $\mathrm{C}_{8}$ component $\left(2^{3} \mathrm{~S}_{1}\right.$, $F=1 / 2-2{ }^{3} \mathrm{P}_{0}$ transition). The frequency stability was sufficient for the laser to stay on resonance over periods of time of one hour or more without any active stabilization.

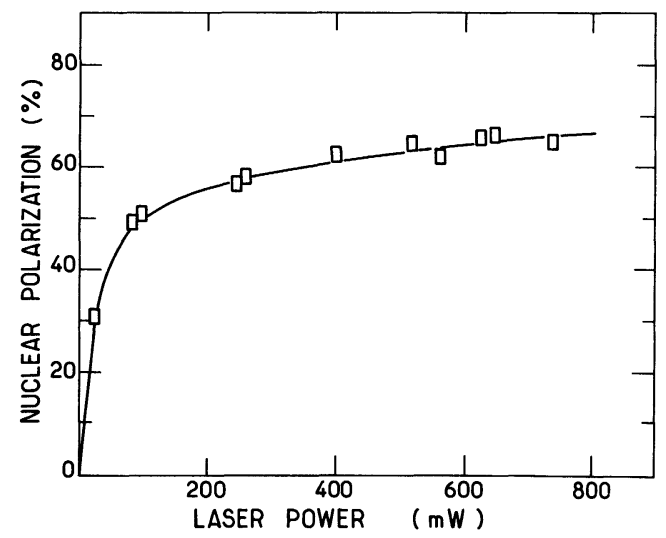

Fig. 5. - Nuclear polarization of a gas of helium-3, obtained in a cell filled with 0.3 torr of gas, as a function of the LNA laser power incident on the cell. The line between the experimental points is just a guide for the eye.

The output power obtained with the present lamp pumped LNA laser at $1.083 \mu \mathrm{m}$ already exceeds the power achieved with previous sources, such as colour centre lasers or LNA lasers pumped by gas lasers. The helium-3 polarization obtained is of the same order (close to $70 \%$ ), as that produced by the colour centre laser [6]. That laser showed a longitudinal mode structure ( 3 modes) which was appropriate for the optical pumping, as well as a clean transverse mode pattern, but was more unstable and certainly much more complicated to run. The present results are comparable or even better than those obtained with a LNA laser pumped by an $\mathrm{Ar}^{+}$laser [15].

We think the present results on optical pumping could be improved further with a more careful adjustment of the circular polarization of the light (this point becomes more and more crucial while the laser power increases, as shown in Ref. [24]), a more detailed study of the influence of the transverse mode structure and finally a more careful elimination of the remaining field gradients in the optical pumping cell. It is also hoped that the output power of the laser can be increased in the near future, which would still result in higher nuclear polarizations of helium-3, as indicated by the still slightly positive slope of the high power end in figure 5 . Actually our present laser operates so close to threshold that even small reductions of the losses in the cavity, such as the improvement of the crystal quality and of the mirror coatings, would result in a significant increase of the laser efficiency. At the same time it is likely that improvements of the quality of the LNA rods will also permit an increase of the pumping power of the lamps, without breaking the crystal. So far, we have operated for months with only one rod at $2.7 \mathrm{~kW}$ of excitation, without any sign of degradation ; nevertheless, with another rod, we tried to increase the excitation level to $3.2 \mathrm{~kW}$ and it broke after a few hours of use only. It is also clear that the excitation of the $\mathrm{Nd}^{3+}$ ions in LNA by the $\mathrm{Kr}^{+}$lamps is not ideal : the absorption profile of the ions [16] does not match the $\mathrm{Kr}^{+}$emission lines very well and the lamps cause much heating for a small contribution to the gain. Two solutions to this problem can be considered. First, there is some hope that a better efficiency of lamp pumped LNA lasers will be achieved with crystals codoped with $\mathrm{Cr}^{3+}$ and $\mathrm{Nd}^{3+}$ [25]. Second, the transverse pumping of LNA rods or slabs with diode arrays emitting at $800 \mathrm{~nm}$ is clearly a promising and realistic possibility for the very near future [26].

\section{Conclusion.}

The lamp pumped LNA laser that we have developed is a compact, reliable and relatively inexpensive source of light at $\lambda=1.083 \mu \mathrm{m}$. It can polarize helium atoms at least as well as previous light sources and seems to have good potentialities for further improvements, including an upscaling of the size of the laser and of the amount of the gas which can be polarized by optical pumping. 
Acknowledgments.

One of us (C.G.A.) gratefully acknowledges finan- cial support from the Swedish Academy of Engineering Sciences in Finland. C. Larat acknowledges support from Thomson-CSF for this work.

\section{References}

[1] Colegrove F. D. and Franken P. A., Phys. Rev. 119 (1960) 680.

[2] Keyer A. R., Rice J. A. and Schearer L. D., J. Geophys. Res. 66 (1961) 4163.

[3] Colegrove F. D., Schearer L. D. and Walters G. K., Phys. Rev. 132 (1963) 2561.

[4] Daniels J. M. and Timsit R. S., Can. J. Phys. 49 (1971) 525, 539.

[5] For a review, see for instance, BETTS D.S. and LeduC M., Ann. Phys. Fr. 11 (1986) 267.

[6] NAcher P.-J., Leduc M., Trénec G. et Laloë F., J. Phys. Lett. France 43 (1982) L-525.

[7] Lhuillier C. and LAloË F., J. Phys. France 40 (1979) 239.

[8] See for instance : Workshop on polarized ${ }^{3} \mathrm{He}$ beams and targets, A.I.P. Proc. $\mathrm{n}^{\circ} 131$ (New York, 1985) and included references.

[9] Leduc M., Crampton S. B., Nacher P.-J. and LALOË F., Nucl. Sci. Appl. 2 (1984) 1.

[10] Milner R. G., MCKeown R. D. and Woodward C. E., Nucl. Instrum. Methods A 257 (1987) 286.

[11] Timsit R. S., Daniels J. M., Dennig E. I., KIANG A. K. C. and MAY A. D., Can. J. Phys. 49 (1971) 508.

[12] Passell L. and Schermer R. I., Phys. Rev. 150 (1966) 146.

Williams W. G., Nukleonika 25 (1980) 769.

[13] Kulsrud R. M., Furth H. P., Valeo E. J. and Goldhaber M., Phys. Rev. Lett. 49 (1982) 1248.
[14] Gray L. G., Giberson K. W., Chu-Cheng, KeIFFER R. S., DUNNING F. B. and WALTERS G. K. Rev. Sci. Instrum. 54 (1983) 271.

[15] Daniels J. M., Schearer L. D., Leduc M. and NACHER P.-J., J. Opt. Soc. Am. B 4 (1987) 1133.

[16] Schearer L. D., Leduc M., Vivien D., LeJus A.M. and THERY J., I.E.E.E. J. Quantum Electron. QE 22 (1986) 713.

[17] Aubert J.-J., Wyon C., Cassini A., HaRdy V. and Hamel J., Opt. Commun. 69 (1989) 299.

[18] Bohler C. L., Schearer L. D., LeduC M., NACHER P.-J., ZACHOROWSKI L., MILNER R. G., MCKEOWN R. D. and WOODWARD C. E., J. Appl. Phys. 63 (1988) 2497.

[19] MILNER R., private communication.

[20] ViVIEN D. et LeJUS A.-M., private communication.

[21] Koechner W., Appl. Opt. 9 (1970) 1429 and 2548.

[22] Metcalf D., De GiovanNi, P. Zachorowski J. and Leduc M., Appl. Opt. 26 (1987) 4508.

[23] Pinard M. and Van Der Linde J., Can. J. Phys. 52 (1974) 1615.

[24] Timsit R. S. and Daniels J. M., Can. J. Phys. 49 (1971) 545.

[25] Viana B., Ponçon V., Garapon C., Lejus A.-M., Vivien D. et Boulon G., J. Phys. Colloq. France 48 (1987) C7-505.

[26] Allik T. H., Hovis W. W., Caffey D. P., King V., Opt. Lett. 14 (1989) 116. 